\title{
Recuperação de áreas campestres: pesquisas para conduzir à recuperação ecológica
}

A carência de informações sobre a propagação de espécies nativas é uma das principais justificativas para o não atendimento à legislação vigente ou para a utilização de espécies exóticas, em ambientes campestres após a realização de atividades econômicas, que exigem a recuperação da área degradada. O presente trabalho apresenta uma revisão comparativa sobre os estudos conduzidos em campos de altitude (CA) e em campos rupestres (CR), levantando informações quanto a propagação das espécies com maior ocorrência, as quais possam subsidiar projetos de recuperação. Verificou-se que a maior parte do conhecimento produzido é focado nos $C R$, mais especificamente voltados à ecologia e descrição de novas espécies. Dentre as espécies de maior frequência em levantamentos sobre CA observou-se que Achyrocline satureioides, Clethra scabra, Coccocypselum condalia, Fuchsia regia e Casearia decandra, apresentam informações básicas para sua condução em projetos de recuperação; as espécies Leptostelma maximum, Peperomia galioides, Polygala paniculata, Siphocampylus westinianus e Verbesina glabrata, são pouco estudadas. As demais espécies avaliadas apresentam conhecimento ecológico que deve ser explorado, antes de sua utilização em campo. Na medida em que os CA são explorados e sua recuperação é obrigatória, deve ser ampliado o conhecimento existente para esta fitofisionomia, naquilo em que há carência de informação científica.

Palavras-chave: Campos de altitude; Campos rupestres; Estratégia de sobrevivência; Regeneração natural; Recuperação áreas de degradadas.

\section{Recovery of field areas: researches to lead ecological recovery}

The lack of information on the propagation of native species is one of the main reasons for not complying with current legislation or for the use of exotic species, in rural environments after carrying out economic activities, which perform the recovery of the degraded area. This work presents a comparative review between the studies led on altitude fields (CA) and the rupestrian fields (CR), providing information about the structure and dynamics of the vegetation to support projects of recovery. Was found that most of the knowledge produced is focused on the CR, but specifically focused on the ecology and description of new species. Among the species of higher frequency on the CA, was observed that Achyrocline satureioides, Clethra scabra, Coccocypselum condalia, Fuchsia regia e Casearia decandra, present basic information to be included in a recovery project; contrary to Leptostelma maximum, Peperomia galioides, Polygala paniculata, Siphocampylus westinianus and Verbesina glabrata, which are little studied. The other assessed species have ecological knowledge that must be explored before their use in field. To the extent that increase exploited of CA their recovery is mandatory, thus the knowledge about these phytophysiognomies needs to be expanded, where there is a lack of scientific information.

Keywords: Altitude fields; Natural regeneration; Recovery of degraded areas; Rocky fields; Survival strategy.

Topic: Desenvolvimento, Sustentabilidade e Meio Ambiente

Reviewed anonymously in the process of blind peer.
Received: $\mathbf{1 1 / 0 7 / 2 0 2 0}$

Approved: 20/10/2020
Railma Pereira Moraes (iD

Instituto Federal do Amazonas, Brasil

http://lattes.cnpq.br/2492271406076604

http://orcid.org/0000-0002-9543-8448

railmoraes@yahoo.com.br

Rossi Allan Silva (iD)

Universidade Federal de Lavras, Brasil http://lattes.cnpq.br/1838509104252299 http://orcid.org/0000-0001-8655-8571 rossiallan@gmail.com

José Aldo Alves Pereira (iD

Universidade Federal de Lavras, Brasil http://lattes.cnpq.br/3542993477950454

http://orcid.org/0000-0001-6602-0504

j.aldo@dcf.ufla.br

\author{
Warley Augusto Caldas Carvalho (iD \\ Universidade Federal de São João Del-Rei, Brasil \\ http://lattes.cnpq.br/8343479823138548 \\ http://orcid.org/0000-0002-1524-1531 \\ wacaldas@yahoo.com.br \\ Kelly Tatiana Bocanegra González (ic) \\ Universidade do Tolima, Brasil \\ http://lattes.cnpq.br/4704818704945940 \\ http://orcid.org/0000-0001-7177-5856 \\ ktbocanegrag@gmail.com
}

Referencing this:

MORAES, R. P.; SILVA, R. A.; PEREIRA, J. A. A.; CARVALHO, W. A. C.; GONZÁLEZ, K. T. B.. Recuperação de áreas campestres: pesquisas para conduzir à recuperação ecológica. Natural Resources, v.10, n.3, p.122137, 2020. DOI: http://doi.org/10.6008/CBPC22379290.2020.003.0013 


\section{INTRODUÇÃO}

Os padrões de uso da terra têm influência direta nos processos naturais e ecológicos, sendo a causa de graves distúrbios a tal ponto que a recuperação da estrutura e funcionalidade da comunidade vegetal passa a necessitar de intervenções humanas sistemáticas. A partir das atividades de recuperação pode-se permitir o retorno do sistema afetado, a um estado satisfatório e a continuidade dos serviços ecossistêmicos que o meio ambiente oferece (TURNER et al., 1997; SANSEVERO et al., 2017). Com a crescente demanda por recursos naturais e o modelo econômico orientado para a extração, a exemplo da mineração (RAMIREZVILLEGAS et al., 2014), áreas sensíveis vêm sendo exploradas. Visando a conservação e proteção, a legislação do código florestal recomenda o plantio preferencial de espécies nativas, com as mesmas características ecológicas daquelas anteriores as atividades de exploração (Lei Federal no 12.65/2012) (BRASIL, 2012).

As plantas nativas são muitas vezes melhores em termos de sobrevivência, crescimento e reprodução em locais de mineração do que plantas introduzidas (YOON et al., 2006). Contudo, a carência de informações sobre a propagação de espécies nativas é uma das principais justificativas para o não atendimento à legislação ou para a utilização de espécies exóticas. Sobre a vegetação campestre, tal carência dá-se pelo fato de não apresentarem rendimento lenhoso significativo, que até um passado recente, era usual os órgãos ambientais autorizarem a conversão para uso alternativo do solo com poucas restrições de conservação (VASCONCELOS, 2014). Atualmente é reconhecida a importância dos campos e suas inúmeras funções abióticas, tais como, áreas de recarga de aquífero, regularizadoras e filtradoras da vazão em cabeceiras de surgências nos ambientes de altitude (SAFFORD, 1999; VASCONCELOS, 2011), além de guardarem espécies relíquitos, endêmicas (SAFFORD, 1999) e outras com potencial ornamental, bem como medicinal também.

Nos últimos anos, a diversidade funcional tem sido usada em ecologia como critério para a seleção de espécies em ambientes degradados por meio de análises de tipos funcionais de plantas (CASANOVES et al., 2011). O princípio desta metodologia é a validação de traços funcionais que são definidos como os componentes morfológicos, fisiológicos, bioquímicos, estruturais, fenológicos ou ainda as características comportamentais das plantas que são expressas em fenótipos, em resposta às propriedades e aos processos ecossistêmicos (KATTGE et al., 2011). Assim, a diversidade funcional pode contribuir para a composição de grupos de plantas em diferentes estágios sucessionais. Tal estratégia foi adotada por Bocanegra-González et al. (2015), estabelecendo tipos diferentes de plantas para a recuperação de ecossistemas de florestas tropicais úmidas.

Neste contexto, o presente trabalho tem como objetivo apresentar os estudos conduzidos sobre os campos de altitude (CA) e campos rupestres (CR), levantando informações existentes acerca da estrutura e da dinâmica da vegetação de CA, bem como propor tipos funcionais de plantas com características adequadas para os diferentes estágios sucessionais, com vistas a refinar as informações sobre os processos de recuperação de áreas degradas com destaque para novas metodologias. A proposta é responder aos seguintes questionamentos: As informações produzidas pela ciência são suficientes para subsidiar projetos de recuperação em CA? Quais espécies presentes nos CA podem apresentar informações suficientes para 
subsidiar o seu uso em projetos de recuperação de áreas degradadas ou alteradas? E, por fim, espera-se promover e incentivar maiores discussões sobre a eficiência dos projetos de recuperação destas áreas, ampliando os estudos sobre diversidade de espécies vegetais ao longo do processo de restauração ecológica.

\section{MATERIAIS E MÉTODOS}

Primeiramente foram abordados conceitos essenciais para distinguir os CA e CR, assim como conhecer os principais métodos de recuperação em ambientes campestres de altitude e a legislação empregada no Brasil (Resolução CONAMA n 423/2010).

A pesquisa foi subdividida em duas partes, sendo a primeira voltada à comparação dos estudos desenvolvidos nos CA e CR, a segunda aborda as espécies que apresentam maior frequência nos levantamentos dos CA, conduzindo dessa forma para as análises dos tipos funcionais de plantas. Finalmente, em cada grupo funcional foram identificadas as espécies com maior número de informações reprodutivas para fortalecer os processos de propagação e estabelecimento em projetos de recuperação de áreas degradadas.

\section{Pesquisa comparativa: Campos de Altitude (CA) e Campos Rupestres (CR)}

Neste estudo foram utilizados dados disponíveis na base SCOPUS até 20 de dezembro de 2017. Para encontrar publicações sobre CR buscou-se pelos termos 'campo rupestre' e 'campos rupestres', e para CA 'campos de altitude', 'campo de altitude' e 'high-altitude fields'. Primeiramente, foi analisada a evolução, observando para cada paisagem, o número de publicações por ano, nos últimos 10 anos.

Na sequência, foram avaliadas as publicações dos 10 últimos anos dos CA e comparadas às publicações do ano de 2017 sobre CR. Tal comparação deu-se para evitar disparidade, pois, no período de 10 anos, enquanto as publicações de CR somam 396, em CA são apenas 63. Cada estudo foi classificado de acordo com a área de estudo (botânica, taxonomia, conservação, ecologia, fisiologia, nova espécie, propagação de sementes e outros); e a universidade de origem do primeiro autor.

\section{Espécies com potencial para a recuperação nos campos de altitude}

Foram avaliados seis levantamentos botânicos de CA do sudeste brasileiro. O banco de dados foi alimentado com artigos (CAIAFA et al., 2005; REZENDE et al., 2013; SALIMENA et al., 2013; MEIRELES et al., 2014; GIOVANETTI-ALVES et al., 2016) e uma tese (BARROS, 2014), mas foram desconsiderados levantamentos voltados tão somente a uma ou algumas famílias. Com os dados formatados foram excluídas espécies com identificação somente ao nível do gênero.

Com base no estudo comparativo dos seis levantamentos botânicos citados foram selecionadas as espécies frequentes em no mínimo três levantamentos, totalizando 41 espécies. Destas, foram avaliados os traços funcionais acerca do estágio de sucessão, tipo de fruto, síndrome de dispersão, forma de vida, endêmica ou não, disponíveis nos bancos de dados no site Flora do Brasil, florasbs, tropics.org, e nos trabalhos de Barbosa et al. (2015) e Peres (2016). 


\section{Tipos Funcionais de Plantas (TFP)}

Organizada a matriz das 41 espécies e de seus traços funcionais, gerou-se a Análise Hierárquicas de Clusters, feita com o método de Ward, conforme Bocanegra-González (2015). Para o cálculo da distância, utilizou-se metodologia proposta por Gower (1971), que utiliza a distância de Jaccard, pois trata-se de variáveis qualitativas. As análises foram feitas com o software Infostat (DI RIENZO et al., 2008).

Avaliou-se os tipos funcionais conforme as características das espécies, que foram classificadas a partir dos traços funcionais recomendados para os processos de recuperação na fase inicial (composto basicamente por ervas de síndrome de dispersão anemocórica e autocórica), intermediário (agrupou espécies pioneiras e não pioneiras, privilegiando aquelas com síndromes de dispersão autocórica e anemocórica, com frutos capsulares) e avançado (incluiu as não pioneiras, com características para atrair e manter a fauna). A classificação deu-se com base em conhecimento pré-estabelecido para os ambientes campestres.

Para cada espécie representada no grupo funcional foram levantadas informações que ajudam a entender a regeneração e posteriormente possam contribuir para a propagação das espécies durante o uso em projetos de recuperação em CA, principalmente após atividade de mineração. Por fim, com base nos levantamentos botânicos e nas informações disponíveis para cada espécie foram indicadas aquelas com maior potencial para ser usada em projetos de recuperação de CA.

\section{RESULTADOS E DISCUSSÃO}

\section{Definições para ambientes campestres: CA e CR}

Vasconcelos (2011) classificou os CA e CR como ambientes distintos. Para este autor os CA referemse aos ambientes campestres sobre granito-gnaisse na Serra da Mantiqueira e na Serra do Mar; enquanto que os CR têm sua ocorrência associada, principalmente, a afloramentos de quartzito, arenito e minério de ferro, tais como as ocorrências na Serra do Espinhaço, no Quadrilátero Ferrífero e na Chapada Diamantina. Assim, a classificação refere-se a fatores ambientais distintos em CR (efeito do macro, meso e micro relevo) e em CA (condicionado pelos afloramentos rochosos) e, portanto, não mutuamente excludentes (VASCONCELOS, 2014).

Apesar das diferenças edáficas e geológicas, estes dois ambientes apresentam semelhanças fitofisionômicas, inclusive compartilhando alguns táxons vegetais, contudo devem ser considerados distintos (VASCONCELOS, 2011). Dentre outras diferenças, vale ressaltar que se encontram em ambientes fitogeográficos diferentes, os CR estão mais relacionados em áreas de transição entre o Cerrado e a Caatinga, enquanto os CA com a região da Mata Atlântica (SAFFORD, 1999; CAIAFA et al., 2005; VASCONCELOS, 2011).

O art. 5 da Resolução CONAMA n 10/1993 caracteriza a vegetação dos CA como "vegetação com estrutura arbustiva e/ou herbácea, que ocorre geralmente nos cumes litólicos das serras com altitudes elevadas, caracterizando-se por uma ruptura na sequência natural das espécies presentes nas formações fisionômicas circunvizinhas". As comunidades florísticas próprias dessa vegetação são caracterizadas por 
elevado endemismo. Contudo, a maior dominância de espécies herbáceas (SAFFORD, 1999; MARTINELLI, 2007) tem refletido em poucos estudos, de forma que pouco se conhece sobre as espécies de ocorrência desta fitofisionomia. Deve-se ressaltar ainda que a vulnerabilidade da vegetação, as mudanças climáticas (ASSIS et al., 2016) e as interações com outras matrizes antrópicas da paisagem podem ser muito agressivas para as espécies de CA.

O pastejo intenso e as queimadas podem resultar em aumento na quantidade de ervas daninhas e na diminuição das forragens, afetando significativamente a variação espacial da vegetação de pastagens alpinas (WEN et al., 2013). Possivelmente, estes distúrbios têm afetado a composição florística campestre, ocasionando a presença de espécies exóticas em levantamentos, por exemplo várias espécies de pinus na Serra do Mar (MOCOCHINSKI et al., 2008), bem como Melinis minutiflora (HOFFMANN et al., 2008; NASCIMENTO et al., 2016). Diante do exposto, busca-se adotar práticas mais eficientes de recuperação e conservação de campos, fazendo uso de conceitos específicos dessas áreas (PARROTTA et al., 2001).

\section{Conceitos para recuperação em CA}

O objetivo da ecologia de recuperação de áreas degradadas não é necessariamente restaurar um ambiente para um ideal primitivo e pré-humano, mas uma visão de longo prazo ainda é importante, porque aumenta consistentemente a biodiversidade e os serviços ecossistêmicos (ROBERTS et al., 2009). No Brasil, devido à dificuldade de identificar os níveis de referência em paisagens campestres, criou-se a resolução CONAMA n ${ }^{\circ} 423 / 2010$, que estabelece parâmetros para identificação e análise da vegetação primária e dos estágios sucessionais, estabelecendo critérios para a caracterização de cada estágio de regeneração (inicial, médio e avançado), baseado na vegetação presente nos CA.

Apesar da relevância de se ter uma base para avaliação da regeneração, deve-se considerar que a omissão de espécies em uma determinada caracterização, poderá induzir a uma avaliação errada. Além disso, levantamentos botânicos realizados em diferentes paisagens caracterizadas como CA têm mostrado diferentes composições vegetais (BRADSHAW, 1997; MOCOCHINSKI et al., 2008). De forma que seria perigoso uniformizar as espécies de ocorrência em CA.

Tratando das técnicas de restauração ecológica, observa-se que a estocagem do solo superficial (topsoil) têm apresentado bons resultados, sendo amplamente difundida para atividades de revegetação após mineração de bauxita (BARROS et al., 2012). A técnica consiste no armazenamento do topsoil durante a extração e o uso deste solo sobre o relevo recriado com o término das atividades de mineração (BARROS et al., 2012; MARTINS, 2014). A matéria orgânica, a fauna do solo, fauna de microrganismos e os propágulos de plantas nativas presentes no topsoil facilitam o restabelecimento da cobertura vegetal, além da ciclagem de nutrientes (AQUINO et al., 2009; MACDONALD et al., 2015).

Em outras técnicas de recuperação de campos, visando maximizar as chances de retorno de algumas espécies desejadas ou até mesmo para permitir a rápida cobertura do solo se tem adotado o uso da mistura de sementes, sendo importante a inclusão de sementes de espécies locais (SPOLETO et al., 2013). Os autores recomendam equilíbrio botânico na mistura de gramíneas comerciais, dicotiledôneas não leguminosas e as 
espécies nativas, também reiteram que apesar de resultar em estabilidade mais duradoura, a alta variedade da mistura de nativas terá pequeno efeito sobre a cobertura do solo.

Na Serra do Caparaó a redução do distúrbio humano em altitudes mais elevadas provavelmente resultou em sucessão de floresta em alguns campos inferiores, mas sua extensão atual provavelmente é influenciada por atividades antropogênicas (VERÍSSIMO et al., 2012). Apesar de reconhecer a relevância de tais práticas é importante estabelecer cautela, pois o fogo quando é utilizado com frequência pelo homem, expõe temporariamente o solo, deixando-o vulnerável à erosão e à sedimentação, facilitando a perda de nutrientes, além de beneficiar algumas espécies invasoras (MOCOCHINSKI et al., 2008).

Práticas de recuperação de áreas degradadas podem ser adotadas combinadas, elevando as chances de sucesso dos projetos. Neste caso o custo elevado torna necessário selecionar uma ou algumas medidas de recuperação ecológica, considerando as especificidades e facilidades locais (Liu et al. 2016). Mas seja qual for a prática para a recuperação adotada, bem como para o monitoramento do estágio de sucessão as pesquisas são essenciais para conhecer as espécies que ocorrem em CA, assim como seu potencial de uso em projetos de recuperação de área degradada. Uma das contribuições deste trabalho foi levantar as principais espécies de ocorrência em CA e avaliar aquelas com maior potencial para uso em recuperação, com base em critérios ecológicos e informações disponíveis que ajudaram na utilização destas espécies em projetos de recuperação.

\section{Estudos atuais sobre CA e CR}

Verificou-se que as publicações relacionadas aos CR são numericamente superiores e têm crescido com o passar do tempo, enquanto que as publicações sobre CA são mais constantes, chegando a 13 no ano de 2016. Tais resultados são influenciados pela larga extensão do CR que abrange áreas nos estados da Bahia, Minas Gerais, Rio de Janeiro e São Paulo. As altitudes elevadas dos CA podem ser outro empecilho para as pesquisas. Na Mantiqueira Meridional a altitude pode chegar a $2.800 \mathrm{~m}$ (MOREIRA et al., 1977). Outra causa pode ser a aplicação errônea de denominações usadas para os tipos de vegetação campestre, conforme cita Vasconcelos (2011).

Ao analisar a instituição de origem dos primeiros autores nas publicações verificou-se que os CA possuem predomínio nos estados de Minas Gerais (UFLA=5 e UFMG=5) e do Rio de Janeiro (JBRJ=5 e UFRJ=7). Os grupos que pesquisam em CR parecem mais consolidados nos estados de Minas Gerais (UFMG=12), e na sequência encontram-se os estados da Bahia (UEFS=8) e de São Paulo (UNICAMP=6). Vale ressaltar que publicações sobre CA estão distribuídas ao longo de 10 anos. Assim, diferente do CR as instituições ainda não possuem grupos de trabalhos com pesquisas continuadas.

Ao avaliar o objeto de estudo verifica-se a alta incidência de publicações com a descrição de novas espécies (animais e vegetais), reiterando que as áreas campestres apresentam alto grau de endemismo (SAFFORD, 1999; SCARANO, 2002), ainda com muitas espécies desconhecidas (Tab. 1). 
Tabela 1: Objeto de estudo das publicações sobre campos de altitudes (CA=2008-2017) e os rupestres (CR=2017).

\begin{tabular}{|c|c|c|c|c|}
\hline \multirow{2}{*}{ Objeto de estudo } & \multicolumn{2}{|c|}{ Publicações sobre CA (2008-2017) } & \multicolumn{2}{|c|}{ Publicações sobre CR (2017) } \\
\hline & no & $\%$ & $\mathrm{n} \underline{0}$ & $\%$ \\
\hline Botânica e taxonomia & 10 & 17,54 & 7 & 12,28 \\
\hline Conservação & 11 & 19,30 & 6 & 10,53 \\
\hline Ecologia & 15 & 26,32 & 6 & 10,53 \\
\hline Fisiologia & 2 & 3,51 & 3 & 5,26 \\
\hline Nova espécie & 12 & 21,05 & 13 & 22,81 \\
\hline Outros & 11 & 19,30 & 17 & 29,82 \\
\hline Propagação e sementes & 2 & 3,51 & 5 & 8,77 \\
\hline TOTAL & 63 & & 57 & \\
\hline
\end{tabular}

Corroborando para o pequeno número de publicações sobre CA e CR, observa-se o baixo percentual de estudo sobre a propagação de espécies e estudos sobre as sementes das espécies desses ambientes, visto que espécies campestres podem apresentar inúmeras dificuldades quanto à regeneração (LE STRADIC et al., 2015). Para Silveira et al. (2016) encontrar o conjunto de condições que permitam o retorno das comunidades de plantas nativas é uma tarefa desafiadora na restauração do CR.

\section{Vegetação de CA}

A vegetação é dominada por gramíneas, outras ervas e algumas pteridófitas restritas aos cumes das montanhas do sudeste do Brasil (SAFFORD, 1999). Neves \& Conceição (2010) consideram que pode haver ocorrência dos mesmos táxons nos $\mathrm{CA}$ e $\mathrm{CR}$, devido às condições ambientais semelhantes. As famílias mais ricas ao sul da Serra da Mantiqueira Meridional foram Asteraceae, Fabaceae e Cyperaceae (MEIRELES et al., 2014); enquanto que no Parque Estadual da Serra do Brigadeiro destacam-se Orchidaceae, Asteraceae, Melastomataceae e Cyperaceae (CAIAFA et al., 2005). Nos CA da Serra do Mar Paranaense verificou-se maior riqueza de Asteraceae, Poaceae, Melastomataceae e Cyperaceae (MOCOCHINSKI et al., 2008). Para Stehmann et al. (2009) a família Asteraceae é a mais diversificada nos ambientes campestres.

Enquanto algumas famílias de plantas são compartilhadas pelos CA e CR, diferentes estudos mostram que os CA apresentam enorme diversidade de espécies. No Parque Estadual da Serra do Brigadeiro foram coletadas 81 espécies (CAIAFA et al., 2005), na Serra da Mantiqueira Meridional/MG (MEIRELES, 2014) encontrou-se 129 espécies e em Poços de Caldas-MG foram coletadas 152 espécies (BARROS 2014), sendo comuns nestes ambientes as espécies como Achyrocline alata DC, Achyrocline satureioides (Lam.) DC., assim como espécies do gênero Baccharis e Tibouchina. Os levantamentos botânicos em diferentes paisagens de CA têm mostrado diferentes composições vegetais.

Das 41 espécies mais frequentemente encontradas nos levantamentos botânicos trabalhados nos CA, 21 são classificadas como indicadoras de estágio avançado de sucessão, segundo a resolução CONAMA $n^{\circ}$ 423/2010. O levantamento mostrou a predominância de espécies consideradas não pioneiras, encontrando-se maior equilíbrio quanto à síndrome de dispersão, assim distribuídas: autocórica (13), anemocórica (16) e zoocórica (11). Estas características e a classificação da síndrome de dispersão podem indicar que os campos estudados apresentam estabilidade, tendo em vista que a diversidade é essencial para a funcionalidade do ecossistema. Quanto ao hábito, espécies caracterizadas como ervas (21) foram as mais 
encontradas, justificando-se pela paisagem campestre, onde são encontradas poucas árvores e arbustos (VASCONCELOS, 2014).

\section{Tipos funcionais de plantas}

Os tipos funcionais foram divididos conforme descrição a seguir e a Figura 1.

TFP1: neste grupo estão o 7,31\% das espécies analisadas. Aqui se encontram ervas, pioneiras, com frutos nucoides e cipsela, com dispersão anemocórica, e não endêmicos; TFP2: este grupo constitui-se por 24,4\% das espécies, sendo arbustos, árvores, erva e liana, não endêmicas e endêmicas do Brasil, com frutos tipo bacoide, capsular e drupoide, com dispersão anemocórica, autocórica e zoocórica; TFP3: este grupo contém 9,7\% das espécies, incluso arbustos e árvores, não endêmicas do Brasil, pioneiras, com frutos de tipo cápsula e drupoide, com dispersão anemocórica e zoocórica; TFP4: neste grupo encontram-se 24,5\% das espécies avaliadas. São ervas, subarbustos e arbustos, pioneiras e não pioneiras com frutos tipo capsulas, cipsela, nucoide e sâmara, com síndromes de dispersão autocórica e anemocórica e espécies endêmicas e não endêmicas do Brasil; TFP5: o grupo contém o 14,6\% das espécies. São ervas, pioneiras e não pioneiras, não endêmicas, com frutos tipo capsula e síndrome de dispersão autocórica; TFP6: o grupo tem 19,5\% de espécies, incluso ervas e lianas, não pioneiras, com frutos tipo capsula e bacoide, sendo a dispersão por anemocórica e zoocórica.

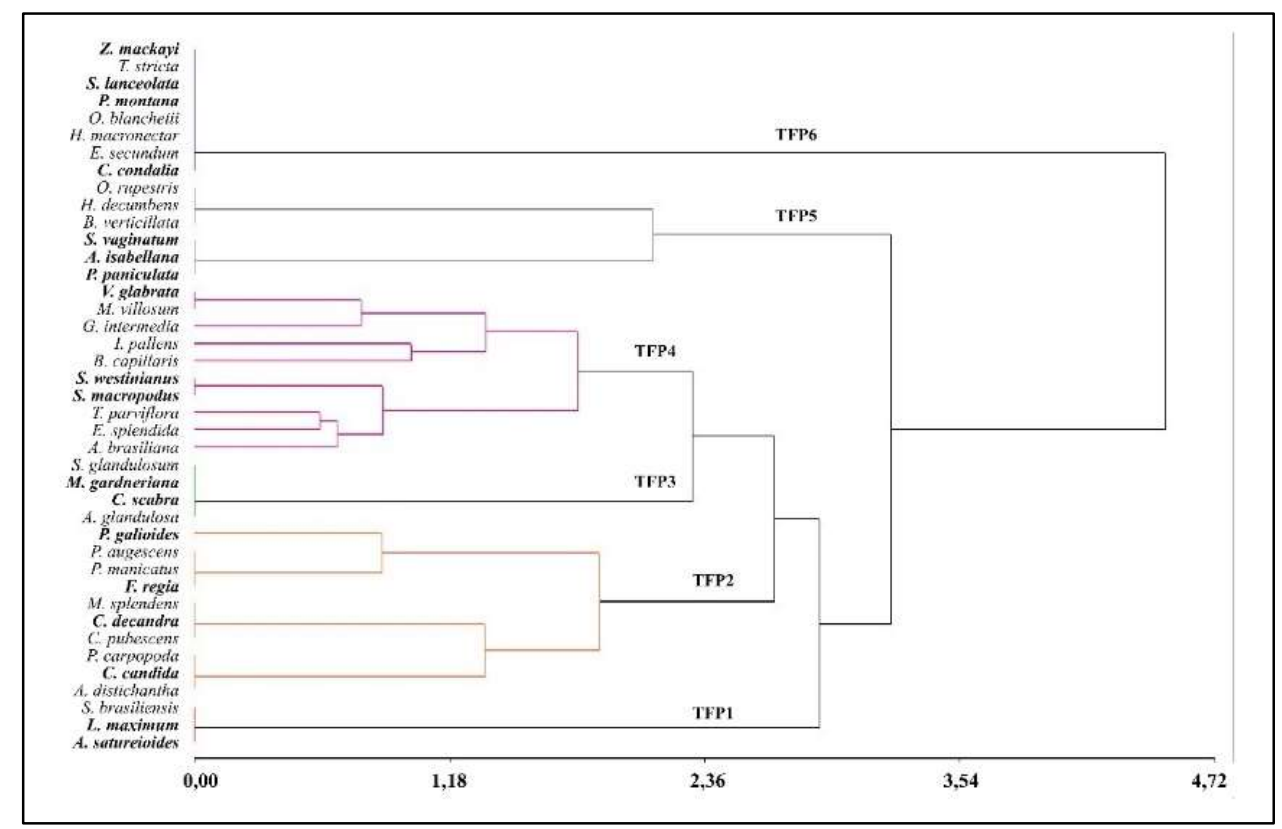

Figura 1: Tipos funcionais de plantas (TFP1, TFP2..., TFP6) para as 41 espécies encontradas nos campos de altitude. Em negrito estão as espécies selecionadas para estudo das informações disponíveis para subsidiar projetos de recuperação de área alterada e degradada.

Pela análise dos traços funcionais as espécies mais frequentes nos CA foram distribuídas em seis tipos funcionais de plantas, isto é, seis grupos com características diferentes que devem ser representados nos processos de restauração do ecossistema em CA. No caso dos TFP1 e TFP5 são recomendados para processos de recuperação iniciais, a exemplo de sítio, com solos muito degradados, onde as espécies destes grupos apresentam características (espécies pioneiras, com dispersão anemocórica e autocórica) que permitem o 
rápido estabelecimento. Por outro lado, os TFP3 e TFP4 são compostos por espécies com diferentes traços funcionais, a fim de estimular o reestabelecimento das espécies e conduzir processos intermediários de recuperação. Finalmente os TFP2 e TFP6 têm espécies com características de ecossistemas mais conservados e são recomendados para áreas com processos intermédios e avançados de recuperação (Fig. 1). Neste último grupo buscou-se agrupar espécies de maior porte, como árvores, mas também, ervas como as orchidaceas, espécies estas que, geralmente, não toleram condições de áreas degradadas.

\section{Espécies com potencial para a recuperação a partir de TFP}

Uma das dificuldades nos trabalhos com espécies campestres é a baixa disponibilidade de encontrar pessoas capacitadas para fazer o reconhecimento ao nível de espécies ou mesmo de gêneros nos CA (VASCONCELOS, 2014). Fato que pode ter excluído algumas espécies de famílias comuns aos campos, como por exemplo: poaceae que apesar de frequentes não apresentavam identificação completa. Esse fato também pode gerar problemas, pois em muitas áreas as espécies são suprimidas sem antes serem identificadas, assim reduzindo as chances de retorno ao ambiente.

Breves descrições das espécies são comumente encontradas na literatura. Contudo para algumas, é possível encontrar a descrição taxonômica e ou chaves para identificação para as espécies: Alstroemeria isabellana (ASSIS, 2012), Coccocypselum condalia (COSTA et al., 2002; PEREIRA et al., 2013), Cranichis candida (MACAGNAN et al., 2011), Fuchsia regia (GRILLO et al., 1998), Leptostelma maximum (HATTORI et al., 2011), Myrsine gardneriana (FREITAS et al., 2015), S. macropodus (SOUZA et al., 2017) Siphocampylus westinianus (GODOY, 1992), Prescottia montana (ABREU et al., 2010; BASTOS et al., 2012). Além disso, alguns sites fornecem a foto para estas espécies em formato digital com imagem de alta resolução.

Além da identificação das espécies dos $C A$ e $C R$, o conhecimento da fenologia reprodutiva das espécies é fundamental para os projetos de recuperação, possibilitando o planejamento da coleta de sementes. Neste sentido, encontrou-se espécies onde foi verificada a existência da presença de flores e frutos em todos os meses do ano, apresentando as fenofases bem distribuídas, como: Alstroemeria isabellana, Sisyrinchium vaginatum e Coccocypselum condalia (Tabela 2). Com base nos resultados a coleta de sementes, entre os meses de fevereiro a junho, compreende o maior número de espécies com frutos.

Para as espécies com ciclo reprodutivo curto deve-se considerar o acompanhamento das fenofases. Há ainda espécies para as quais não foram encontradas publicações científicas sobre a fenologia reprodutiva. Vale ressaltar que as fenofases podem ser diferentes para as mesmas espécies alterando de acordo com as condições ambientais do local (CHUKR, 1992).

Tabela 2: Fenologia reprodutiva das espécies selecionadas, com base em levantamentos botânicos em campos de altitude, no sudeste brasileiro. Legenda: Fl.=floração, Fr.=Frutificação.

\begin{tabular}{|c|c|c|c|c|c|c|c|c|c|c|c|c|c|c|}
\hline \multirow{2}{*}{ TFP } & \multirow{2}{*}{ Espécies } & \multirow{2}{*}{$\mathrm{Fl} / \mathrm{Fr}$. } & \multicolumn{12}{|c|}{ Meses } \\
\hline & & & 1 & 2 & 3 & 4 & 5 & 6 & 7 & 8 & 9 & 10 & 11 & 12 \\
\hline \multirow{3}{*}{1} & A. satureioides & Fl. & & & & & & & & & & & & \\
\hline & (RETTA et al., 2012) & Fr. & & & & & & & & & & & & \\
\hline & C. cândida & Fl. & & & & & & & & & & & & \\
\hline \multirow[t]{2}{*}{2} & (PANSARIN et al., 2017; MACAGNAN et al., 2011) & Fr. & & & & & & & & & & & & \\
\hline & C. decandra & $\mathrm{Fl}$. & & & & & & & & & & & & \\
\hline
\end{tabular}




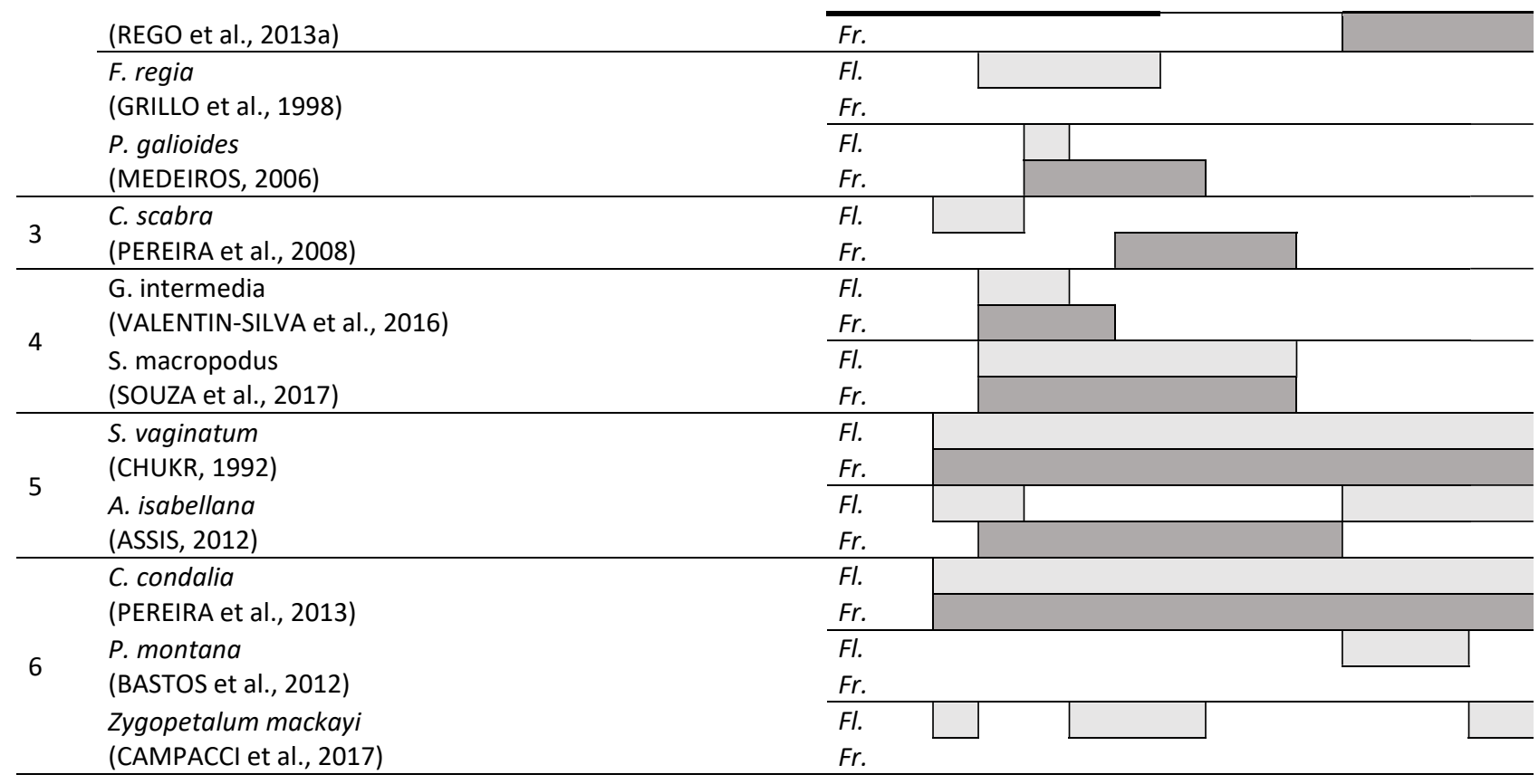

\section{Espécies para uso nos projetos de recuperação}

Para a espécie Achyrocline satureioides Marques \& Barros (2001) e Retta et al. (2012) realizaram estudos para a produção de mudas e obtiveram bons resultados nas formas assexuada ou sexuada. A germinação não possui dificuldades, destacando que o poder germinativo das sementes em local seco aumenta de $68 \%$ para $71 \%$ após seis meses de armazenamento à temperatura ambiente $\left(19-23^{\circ} \mathrm{C}\right)$ (MARQUES et al., 2000; RETTA et al., 2012). Essa espécie também pode ser encontrada em bancos de sementes (MORAES et al., 2017). Para o plantio com semeadura direta, Pellizzaro et al. (2017) citam que Achyrocline satureioides apresentou alta taxa de cobertura do solo, logo no primeiro ano após a semeadura, com produção de sementes na primeira estação chuvosa após o plantio.

Sobre Clethra scabra não foram encontradas informações sobre sua germinação. Contudo, trabalhos de Klein et al. (2009) e Sampaio et al. (2007) citam que a espécie é frequentemente encontrada em ambientes de alta luminosidade com solos pobres ou compactados. Klein et al. (2009) também relatam que a espécie foi encontrada em pilhas de estéreis, em locais onde ocorreu mineração de carvão a céu aberto. Em avaliações de chuva de sementes a espécie mostrou ser abundante e frequente (BEGNINI et al., 2013). Assim, por apresentar abundância de sementes e facilidade de emergência no campo, sua presença em projetos de recuperação poderá ocorrer quando ela estiver próxima a área afetada ou com a introdução de sementes.

Coccocypselum condalia é considerada uma espécie ciófita (VIANA et al., 2014), isto é_intolerante ao sol que necessita de sombra para seu estabelecimento e desenvolvimento. Contudo, por se tratar de uma espécie hemicriptófita (VIANA et al., 2014), suas partes vegetativas podem ser utilizadas durante as etapas de recuperação, desde que não seja área aberta, oferecendo rápida cobertura do solo após distúrbios ambientais (NERI et al., 2011).

Fucsia regia multiplica-se bem por estaquia mas tem sido pouco cultivada no Brasil, embora seja muito valorizada na Europa e América do Norte, onde a espécie tem finalidade ornamental, por causa de suas flores (FALKENBERG et al., 2011). A espécie é relativamente frequente, comumente encontrada próxima 
à clareiras naturais e beiras de rios (FALKENBERG et al., 2011; VILLAGRA et al., 2013), podendo assim, tolerar pequenas alterações ambientais causadas pelo homem.

A Casearia decandra representa uma das poucas árvores levantadas em ambientes campestres, contudo apresenta elevada densidade quando presente no estrato arbóreo de ambientes florestais (NARVAES et al., 2005). Em estudo germinativo verificou-se que a espécie apresenta elevada taxa de germinação (acima de 90\%), em torno de 5 dias, quando suas sementes são expostas a temperaturas de $20^{\circ} \mathrm{C}, 25^{\circ} \mathrm{C}$ e $30^{\circ} \mathrm{C}$, em substrato rolo de papel (HALISKI et al., 2013). Os mesmos autores também fazem a descrição das plântulas e dos frutos, informação essencial para identificação em campo. Rego et al. (2013) realizaram estudo de tolerância à desidratação para as sementes.

Algumas espécies como Myrsine gardneriana ocorrem em maior frequência e densidade em áreas com pequenas alterações antrópicas (SEVEGNANI et al., 2013), isto é, são tolerantes a pequenas perturbações ambientais, sendo indicadas para fases intermediárias e avançadas de recuperação. Enquanto que, Grazielia intermedia e Siphocampylus macropodus são citadas, respectivamente por Valentin-Silva et al. (2016), Souza et al. (2017), como espécies que ocorrem em lugares ensolarados, favorecidas pela alta intensidade de radiação, podendo ser estudadas para uso nas fases iniciais de recuperação.

Durante períodos de estresses ambientais algumas espécies desenvolvem estratégias essenciais à sua regeneração, as quais podem ser entendidas de maneira a maximizar a diversidade vegetal durante o processo de recuperação. O período seco pode causar a mortalidade dos indivíduos de Sisyrinchium vaginatum, enquanto a sua sobrevivência deve-se a preseça expressiva de rafinose que funciona como composto de reserva (CIANCIARUSO et al., 2008), talvez por isso a espécie seja citada como abundante nos bancos de sementes do solo. Espécies como Alstroemeria isabellana que apresentam rizomas, podem regenerar-se por meio destas estruturas após um distúrbio local (ASSIS, 2012). Diante da dificuldade para coleta de sementes viáveis, o estudo de tais estratégias de sobrevivência parece uma oportunidade de aumentar a diversidade das espécies, após a recuperação da área.

Cranichis candida é uma orquídea terrícola e apesar de ser frequentemente encontrada em levantamentos botânicos, não é abundante (RAHAL et al., 2016). Esta espécie, assim como Leptostelma maximum e Peperomia galioides demonstra a capacidade de se adaptar a diferentes condições de umidade e tipos de ambientes (MEIRELES et al., 2014; RAHAL et al., 2016). Entretanto não foram encontradas informações sobre a regeneração da espécie, sendo este conhecimento essencial para projetos de recuperação. Prescottia montana, outra orquídea, mesmo sendo considerada espécie ameaçada no Paraná apresenta poucas informações. Para a família orchidaceae vale ressaltar que algumas espécies terrícolas se adaptam a ambientes perturbados, podendo tornar-se invasoras, enquanto outras são sensíveis a ambientes degradados (RAHAL et al., 2016), manifestando assim, o potencial destas como bioindicador, seja para identificar áreas conservadas ou alteradas.

Em áreas campestres os incêndios são recorrentes podendo atuar como uma força seletiva ou superar a dormência física de algumas sementes (GORGONE-BARBOSA et al., 2016). Muitas espécies se beneficiam com o fogo, como: Achyrocline satureioides, Alstroemeria isabellana, Clethra scabra, 
Cossosypselum condalia, Cranichis candida, Myrsine gardneriana, Peperomia galioides, Prescottia montana, Siphocampylus westinianus e Verbesina glabrata, que foram identificadas por Aximoff et al. (2016) após incêndios. A emergência após o fogo pode representar uma adaptação das espécies campestres, tendo em vista que práticas com o uso do fogo são recorrentes nestes ambientes. Alguns autores consideram a gestão do fogo necessária para a conservação dos campos (FIDELIS et al., 2010; KOLB et al., 2016; TROWBRIDGE et al., 2017). Estes resultados indicam que o fogo, quando possível pode ser usado como estímulo a germinação ou a perpetuação de algumas espécies campestres.

Para as espécies Leptostelma maximum, Peperomia galioides, Polygala. paniculata, Siphocampylus westinianus e Verbesina glabrata estudos são necessários para ampliar o conhecimento das mesmas. Embora estas espécies sejam frequentes em levantamentos (CAIAFA et al., 2005; REZENDE et al., 2013; SALIMENA et al., 2013; MEIRELES et al., 2014; GIOVANETTI-ALVES et al., 2016) não foram encontradas informações relevantes para a propagação e regeneração em projetos de recuperação. $O$ presente estudo reconhece que estão sendo realizados diferentes estudos com regeneração e propagação de outras espécies campestres (por exemplo: BARBOSA et al., 2014; LE STRADIC et al., 2014; SILVEIRA et al., 2014), contudo faz-se necessário o estabelecimento de estratégias de conservação para as áreas de $C A$, tendo em vista que, mesmo para as espécies mais frequentes há falta de informações essenciais para a recuperação ecológica do ambiente. Vale ressaltar que várias espécies presentes nos CA apresentam potencial econômico tais como a coleta de sementes, produção de mudas, coleta e produção de flores (Alstroemeria isabellana, Fuchsia regia), medicinal (Achyrocline satureioides, Polygala paniculata), dentre outras.

Para a escolha de espécies destinadas à recuperação de áreas alteradas ou degradadas, Pinheiro et al. (2007) consideram que além dos indicadores ecológicos, deva-se avaliar o potencial biótico de cada uma com base na produção e germinação de sementes, no estabelecimento da produção de mudas, em pesquisas de viveiro e em silvicultura de plantações. Com base nas informações atuais para as espécies estudadas (recuperação inicial), deve ser avaliado o uso de Achyrocline satureioides (TFP1). Para os processos intermediários de recuperação as espécies estudadas que demonstraram potencial de uso foram Clethra scabra e Myrsine gardneriana (TFP3). Em casos de ecossistemas menos impactados, onde o processo de recuperação encontra-se entre o intermediário e o avançado, as espécies Fuchsia regia, Casearia decandra (TFP2) e Coccocypselum condalia (TFP6) são recomendadas. Por fim, para ampliar a diversidade de espécies maiores estudos são necessários.

\section{CONCLUSÕES}

Verificou-se que a maior parte do conhecimento produzido é focado nos Campus Rupestres, mais especificamente voltados à ecologia e descrição de novas espécies. As espécies de maior frequência em levantamentos sobre Campus de Altitude, observou-se que Achyrocline satureioides, Clethra scabra, Coccocypselum condalia, Fuchsia regia e Casearia decandra, apresentam informações básicas para sua condução em projetos de recuperação; as espécies Leptostelma maximum, Peperomia galioides, Polygala 
paniculata, Siphocampylus westinianus e Verbesina glabrata, são pouco estudadas. As demais espécies avaliadas apresentam conhecimento ecológico que deve ser explorado, antes de sua utilização em campo.

\section{REFERÊNCIAS}

ABREU, N. L.; MENINI NETO, L.. As subfamílias Vanilloideae e Orchidoideae (Orchidaceae) em um fragmento da Serra da Mantiqueira, Minas Gerais, Brasil. Boletim de Botânica da Universidade de São Paulo, São Paulo, n.28, v.1, p.15-33, 2010.

AQUINO, C.; BARBOSA, L. M.. Classes sucessionais e síndromes de dispersão de espécie arbóreas e arbustivas existentes em vegetação ciliar remanescente (Conchal, SP), como subsídio para avaliar o potencial do fragmento como fonte de propágulos para enriquecimento de áreas revegetadas no Rio Mogi-Guaçu, SP. Revista Árvore, Viçosa, n.33, p.349-358, 2009.

ASSIS, M. C.. Alstroemeriaceae na Região Sul do Brasil. Rodriguésia, n.63, p.1117-1132, 2012.

ASSIS, M. V.; MATTOS, E. A.. Vulnerabilidade da vegetação de campos de altitude às mudanças climáticas. Oecologia Australis, n.20, p.24-36, 2016. DOI: http://doi.org/10.4257/oeco.2016.2002.03

AXIMOFF, I.; NUNES-FREITAS, A. F.; BRAGA, J. M. A.. Regeneração natural pós-fogo nos campos de altitude no parque nacional do Itatiaia, sudeste do Brasil. Oecologia Australis, n.20, p.62-80, 2016. DOI: http://doi.org/10.4257/oeco.2016.2002.05

BARBOSA, B. D. C.; CAPPI, V. S.; RIBEIRO, S. P.; FERNANDES, G. W.. Avaliação da capacidade de rebrotamento pósdistúrbio das plantas lenhosas típicas dos campos rupestres. Ecologia Austral, n.24, p.350-355, 2014.

BARBOSA, L. M.; SHIRASUNA, R. T.; LIMA, F. C.; ORTIZ, P. R. T.. Lista de espécies indicadas para Restauração Ecológica para diversas regiões do Estado de São Paulo. In: SIMPÓSIO DE RESTAURAÇÃO ECOLÓGICA. Anais. 2015. p.303436. DOI: http://doi.org/10.1017/CBO9781107415324.004

BARROS, D. A.; GUIMARAES, J. C. C.; PEREIRA, J. A. A.; BORGES, L. A. C.; SILVA, R. A.; PEREIRA, A. A. S.. Characterization of the bauxite mining of the Poços de Caldas and its socio-environmental impacts. Revista Escola de Minas, n.65, p.127-133, 2012.

BARROS, D. A.. Campos de altitude sob interferência da mineração de bauxita no planalto de Poços de Caldas, MG. Tese de Doutorado. Universidade Federal de Lavras, Lavras, 2014.

BASTOS, C. A.; VAN DEN BERG, C.. A família Orchidaceae no município de Morro do Chapéu, Bahia, Brasil. Rodriguésia, n.63, p.883-927, 2012. DOI: http://doi.org/10.1590/S217578602012000400010

BEGNINI, R.; CASTELLANI, T.. Seed rain under the canopies of female and male Myrsine coriacea, a pioneer tree from the Brazilian Atlantic forest. Journal of Tropical Ecology, n.29, p.391-399, 2013. DOI: http://doi.org/10.1017/S0266467413000400
BOCANEGRA-GONZÁLEZ, K. T.; FERNÁNDEZ-MÉNDEZ, F.; GALVIS-JIMÉNEZ, J. D.. Grupos funcionales de árboles en bosques secundarios de la región Bajo Calima (Buenaventura, Colombia). Bol. Cient. Mus. Hist. Nat, n.19, p.17-40, 2015.

BRADSHAW, A.. Restoration of mined lands: using natural processes. Ecological Engineering, n.8, p.255-269, 1997. DOI: http://doi.org/10.1016/S0925-8574(97)00022-0

BRASIL. Lei n. 12.651, de 25 de maio de 2012. Dispõe sobre a proteção da vegetação nativa. Brasília: DOU, 2012.

CAIAFA, A. N. F.; SILVA, A. F.. Composição florística e espectro biológico de um campo de altitude no Parque Estadual da Serra do Brigadeiro, Minas Gerais. Rodriguésia, n.56, v.87, p.163-173, 2005.

CASANOVES, F.; PLA, L.; DI RIENZO, J. A.. Valoración y análisis de la diversidad funcional y su relación con los servicios ecosistémicos. Turrialba: CATIE, 2011.

CHUKR, N. S.. Flora da Serra do Cipó, Minas Gerais: Iridaceae. Boletim de botânica da Universidade de São Paulo, São Paulo, n.13, p.111-131, 1992.

CIANCIARUSO, M. V.; BATALHA, M. A.. A year in a Cerrado wet grassland: a non-seasonal island in a seasonal savanna environment. Brazilian Journal of Biology, n.68, v.3, p.495501, 2008.

COSTA, C. B.; MAMEDE, M. C. H.. Sinopse do gênero Coccocypselum P. Browne (Rubiaceae) no estado de São Paulo, Brasil. Biota Neotropica, n.2, v.1, p.1-14, 2002.

CONAMA. Conselho Nacional de Meio Ambiente. Resolução n. 423, de 12 de abril de 2010. Dispõe sobre parâmetros básicos para identificação e análise da vegetação primária e dos estágios sucessionais da vegetação secundária nos Campos de Altitude associados ou abrangidos pela Mata Atlântica. Brasília: DOU, 2010.

DI RIENZO, J. A.; CASANOVES, F.; BALZARINI, M. G. GONZÁLEZ, L.; TABLADA, M.; ROBLEDO, C. W.. Software InfoStat. Buenos Aires: Grupo InfoStat, FCA, Universidad Nacional de Córdoba, 2008

FALKENBERG, D. B.. Fuchsia regia. In: CORADIN, L.; SIMINSKI, A.; REIS, A.. Espécies nativas da flora brasileira de valor econômico atual ou potencial plantas para o futuro Região Sul. MMA, 2011. p.764-768.

FIDELIS, A.; DELGADO-CARTAY, M. D.; BLANCO, C. C.; MÜLLER, S. C.; PILLAR, V. D.; FADENHAUER, J. P.. Fire intensity and severity in Brazilian Campos grasslands. Interciencia, v.35, n.10, p.739-745, 2010.

FREITAS, M. D. F.; KINOSHITA, L. S. Myrsine (MyrsinoideaePrimulaceae) no sudeste e sul do Brasil. Rodriguesia, n.66, 
v.1, p.167-189, 2015. DOI: http://doi.org/10.1590/2175$\underline{7860201566109}$

GIOVANETTI-ALVES, R.; ZAÚ, A. S; OLIVEIRA, R. R.. Flora dos Campos de Altitude em quatro áreas do Maciço do Itatiaia, nos Estados do Rio de Janeiro e de Minas Gerais, Brasil. Pesquisas Botânica, n.69, p.109-140, 2016.

GODOY, S. A. P.. Flora da serra do Cipó, Minas Gerais: Campanulaceae. Boletim de Botânica da Universidade de São Paulo, n.13, p.241-257, 1992.

GORGONE-BARBOSA, E.; PIVELLO, V. R.; BAEZA, M. J.; FIDELIS, A.. Disturbance as a factor in breaking dormancy and enhancing invasiveness of African grasses in a Neotropical Savanna. Acta Botanica Brasilica, n.30, p.131137, 2016. DOI: http://doi.org/10.1590/010233062015abb0317

GOWER, J. C.. A general coefficient of similarity and some of its properties. Biometrics, n.27, p.857-874, 1971.

GRILLO, A. A. S.; GIULIETTI, A. M.. Flora da serra do Cipó, Minas Gerais: Onagraceae. Boletim de Botânica da Universidade de São Paulo, São Paulo, n.17, p.109-114, 1998.

HALISKI, S. L.; COSMO, N. L.; GOGOSZ, A. M.; REGO, S. S.; NOGUEIRA, A. C.; KUNIYOSHI. Y. S.. Caracterização morfológica de frutos, sementes, plântulas e germinação de sementes de $C$. decandra. Pesquisa Florestal Brasileira, n.33, p.253-259, 2013. DOI: http://doi.org/10.4336/2013.pfb.33.75.451

HATTORI, E. K. O.; NAKAJIMA, J. N.. A família Asteraceae na Reserva Ecológica do Panga, Uberlândia, Minas Gerais, Brasil. Hoehnea, n.38, p.165-214, 2011.

HOFFMANN, W. A.; HARIDASAN, M.. The invasive grass, Melinis minutiflora, inhibits tree regeneration in a Neotropical savana. Austral Ecology, n.33, p.29-36, 2008. DOI: http://doi.org/10.1111/j.1442-9993.2007.01787.x

KATTGE, J.; DIAS, S.. TRY: a global database of plant functional traits. Global Change Biology, Oxford, n.17, p.2905-2935, 2011.

KLEIN, A. S.; CITADINI-ZANETTE, V.; LOPES, R. P.; SANTOS, R. Regeneração natural em área degradada pela mineração de carvão em Santa Catarina, Brasil. Rem: Revista Escola de Minas, n.62, n.297-304, 2009. DOI: http://doi.org/10.1590/S0370-44672009000300007

KOLB, R. M., PILON, N. A. L.; DURIGAN G.. Factors influencing seed germination in Cerrado grasses. Acta Botanica Brasilica, n.30, p.87-92, 2016. DOI: http://doi.org/10.1590/0102-33062015abb0199

LE STRADIC, S.; SILVEIRA, F. A. O.; BUISSON, E.; CAZELLES, K.; CARVALHO, V.; FERNANDES, G. W.. Diversity of germination strategies and seed dormancy in herbaceous species of campo rupestre grasslands. Ecological Society of Australia, n.40, p.537-546, 2015.

LE STRADIC, S.; BUISSON, E.; NEGREIROS, D.; CAMPAGNE, P.; WILSON FERNANDES, G.. O papel das espécies lenhosas nativas na restauração de Campos Rupestres em pedreiras. Applied Vegetation Science, n.17, p.109-120 2014. DOI: https://doi.org/10.1111/avsc.12058

LIU, Y.; ZHANG, D.; ZHANG, Y.; SHI, M.; SHANG, Z.; HE, L.. Evaluation of restoration effect in degraded alpine meadow under different regulation measures. Transactions of the Chinese Society of Agricultural Engineering, n.32, p.268275, 2016.

MACAGNAN, T. A.; SMIDT, E. C.; AZEVEDO, C. O.. Subtribo Cranichidinae Lindl. (Orchidaceae) no Estado do Paraná, Brasil. Rev. Bras. Bot., n.34, p.447-461, 2011. DOI: http://doi.org/10.1590/S0100-84042011000300017

MACDONALD, S.E.; LANDHAUSSER, S. M.; SKOUSEN, J.; FRANKLIN, J.; FROUZ, J.; HALL, S.; JACOBS, D. F.; QUIDEAU, S.. Forest restoration following surface mining disturbance: challenges and solutions. New Forests, n.46, p.703-732, 2015.

MARQUES, F. C.; BARROS, I. B. I.. Qualidade de sementes de marcela (A. satureioides) provenientes de duas populações do Rio Grande do Sul. Ciência Rural, n.30, p.241-247, 2000.

MARQUES, F. C.; BARROS, I. B. I.. Crescimento inicial de marcela (Achyrocline satureioides) em ambiente protegido. Ciência Rural, n.31, p.517-518, 2001.

MARTINELLI, G.. Mountain biodiversity in Brazil. Revista Brasileira de Botânica, n.30, p.587-597, 2007.

MARTINS, S. V.. Recuperação de matas ciliares: no contexto do novo código florestal. 3 d. Viçosa: Aprenda fácil, 2014.

MEIRELES, L. D.; KINOSHITA, L. S.; SHEPHERD, G. J. Composição florística da vegetação altimontana do distrito de Monte Verde (Camanducaia, MG), Serra da Mantiqueira Meridional, Sudeste do Brasil. Rodriguésia, n.65, v.4, p.831859, 2014. DOI: http://doi.org/10.1590/21757860201465403

MOCOCHINSKI, A. Y.; SCHEER, M. B.. Campos de altitude na serra do mar paranaense: aspectos florísticos. Floresta,

Curitiba, v.38, n.4, p.625-640, 2008. DOI:

http://doi.org/10.1590/S0101-81752004000400009

MORAES, R. P.; CARVALHO, W. A. C.; PEREIRA, J. A. A.; NASCIMENTO, G. O.; BARROS, D. A.. Effect of topsoil stockpiling on the viability of seed bank in field phytophysiognomies Campos de Altitude. Cerne, Lavras, n.23, p.339-347, 2017. DOI: http://doi.org/10.1590/01047760201723032340

MOREIRA, A. A. N.; CAMALIER, C.. Relevo. In: Geografia do Brasil. Rio de Janeiro: IBGE, 1977. p.150.

NARVAES, I. S.; BRENA, D. A.; LONGHI, S. J.. Estrutura da regeneração natural em floresta ombrófila mista na Floresta Nacional de São Francisco de Paula, RS. Ciência Florestal, n.15, p.331-342, 2005

NASCIMENTO, G. O.; PEREIRA, J. A. A.; BARROS, D. A.; SILVA, R. A.; CLEITON JOSÉ, A.; FERREIRA, J. B.; OLIVEIRA, S. S.. Propagule emergence in topsoil from a high-altitude field and implications for bauxite mining area restoration. International Journal of Biodiversity and Conservation, n.8, p.310-319, 2016. DOI: 
http://dx.doi.org/10.5897/IJBC2016.1031

NERI, A. V.; SOARES, M. P.; MEIRA NETO, J. A. A.; DIAS. L. E.. Espécies de cerrado com potencial para recuperação de áreas degradadas por mineração de ouro, Paracatu-MG. Revista Árvore, Viçosa, n.35, p.907-918, 2011. DOI: http://doi.org/10.1590/S0100-67622011000500016

NEVES, S. P. S.; CONCEICAO, A. A.. Campo rupestre recémqueimado na Chapada Diamantina, Bahia, Brasil: plantas de rebrota e sementes, com espécies endêmicas na rocha. Acta Botanica Brasilica, n.24, p.697-707, 2010.

PARROTTA, J. A.; KNOWLES, O. H.. Restoring tropical forests on lands mined for bauxite: examples from the Brazilian Amazon. Ecological Engineering, n.17, p.219-239, 2001.

PELLIZZARO, K. F.; CORDEIRO, A. O. O.; ALVES, M.; MOTTA, C. P.. "Cerrado" restoration by direct seeding: field establishment and initial growth of 75 trees, shrubs and grass species. Revista Brasileira de Botânica, n.40, p.681693, 2017. DOI: http://doi.org/10.1007/s40415-017-0371-6

PEREIRA, Z. V.; KINOSHITA, L. S.. Rubiaceae Juss. do Parque Estadual das Várzeas do Rio Ivinhema, MS, Brasil. Hoehnea, n.40, p.205-251, 2013

PERES, M. K.. Estratégias de dispersão de sementes no bioma cerrado: considerações ecológicas e filogenéticas. Tese (Doutorado) - Universidade de Brasília, Brasília, 2016.

PINHEIRO, K. A. O.; CARVALHO, J. O. P.; QUANZ, B.; FRANCEZ, L. M. B.; SCHWARTZ, G.. Fitossociologia de uma área de preservação permanente no leste da Amazônia: indicação de espécies para recuperação de áreas alteradas. Revista Floresta, Curitiba, n.37, p.175-187, 2007.

RAHAL, M. H.; SOUZA-LEAL, T.; MORAES, C. P.. Ecologia e distribuição espacial de orquídeas terrícolas em Floresta Estacional Semidecidual do município de Araras, São Paulo, Brasil. Iheringia. Série Botânica, n.70, p.217-228, 2016.

RAMÍREZ-VILLEGAS, J.; CUESTA, F.; DEVENISH, C.; PERALVO, M.; JARVIS, A.; ARNILLAS, C. A.. Using species distributions models for designing conservation strategies of Tropical Andean biodiversity under climate change. Journal for Nature Conservation, n.22, p.391-404, 2014.

REGO, S. S.; NOGUEIRA, A. C.; MEDEIROS, A. C. S.; PETKOWICZ, C. L. O.; SANTOS, A. F.. Physiological behaviour of Blepharocalyx salicifolius and Casearia decandra seeds on the tolerance to dehydration. Journal of Seed Science, n.35, p.323-330, 2013.

RETTA, D.; DELLACASSA, E.; VILLAMIL, J.; SUÁREZ, S. A. \& BANDONI, A. L.. Marcela, a promising medicinal and aromatic plant from Latin America: A review. Industrial Crops and Products, n.38, p.27-38, 2012. DOI: http://doi.org/10.1016/j.indcrop.2012.01.006

REZENDE, M. G.; LOYOLA ELIAS, R. C.; SALIMENA, F. R. G.; MENINI NETO, L.. Flora vascular da Serra da Pedra Branca, Caldas, Minas Gerais e relações florísticas com áreas de altitude da Região Sudeste do Brasil. Biota Neotropica, n.13, p.201-224, 2013. DOI: http://doi.org/10.1590/S1676$\underline{06032013000400019}$
ROBERTS, L.; STONE, R.; SUGDEN, A.. The Rise of Restoration. Ecology Science, n.325, p.555, 2009. DOI: http://doi.org/10.1126/science.325 555

SAFFORD, H. D.. Brazilian Paramos I. An introduction to the physical environment and vegetation of the campos de altitude. Journal of Biogeography, n.26, p.693-712, 1999.

SALIMENA, F. R. G.; MATOZINHOS, C. N.; ABREU, N. L.; RIBEIRO, J. H. C.; SOUZA, F. S.. Flora fanerogâmica da Serra Negra, Minas Gerais, Brasil. Rodriguésia, n.64, p.1-34, 2013.

SAMPAIO, M. B.; GUARINO, E. S. G.. Efeitos do pastoreio de bovinos na estrutura populacional de plantas em fragmentos de floresta ombrófila mista. Rev. Árvore, n.31, p.1035-1046, 2007.

SANSEVERO, J. B. B.; PRIETO, P. V.; SÁNCHEZ-TAPIA, A.; BRAGA, J. M. A.; RODRIGUES, P. J. F. P.. Past land-use and ecological resilience in a lowland Brazilian Atlantic Forest: implications for passive restoration. New Forests, n.48, p.573-586, 2017. DOI: http://doi.org/10.1007/s11056-0179586-4

SCARANO, F. R.. Structure, function and floristic relationships of plant communities in stressful habitats marginal to the Brazilian Atlantic rain forest. Annals of Botany, n.90, p.517-524, 2002.

SEVEGNANI, L.; VIBRANS, A. C.; GASPER, A. L.. Considerações finais sobre a Floresta Ombrófila Densa e Restinga. In: VIBRANS, A. C.. Inventário florístico florestal de Santa Catarina. 2013. p.275-279.

SILVEIRA, F. A. O.; NEGREIROS, D.; BARBOSA, N. P U.; BUISSON, E.; CARMO, F. F.. Ecology and evolution of plant diversity in the endangered campo rupestre: a neglected conservation priority. Plant and Soil, n.403, p.129-152, 2016. DOI: http://doi.org/10.1007/s11104-015-2637-8

SILVEIRA, F. A. O.; NEGREIROS, D.; RANIERI, B. D.; SILVA, C. A.; ARAÚJO, L. M.. Effect of seed storage on germination, seedling growth and survival of Mimosa foliolosa (Fabaceae): Implications for seed banks and restoration ecology. Tropical Ecology, n.55, p.385-392, 2014.

SOUZA, G. A.; GODOY, S. A. P.; SALIMENA, F. R. G.. Campanulaceae da Serra Negra, Minas Gerais, Brasil. Rodriguesia, n.68, p.503-513, 2017. DOI: http://doi.org/10.1590/2175-7860201768214

SPOLETO, P.; TOSCA, A.; MARIANNA, G. D.; GUSMEROLI, F.; PECETTI, L.; ROMANI, M.. Comparison of Seed Mixtures for Technical Revegetation at High Altitude. In: BARTH, S.; MILBOURNE, D.. Breeding strategies for sustainable forage and turf grass improvement. Springer, 2013. p.255-260. DOI: http://doi.org/10.1007/978-94-007-4555-1 33

STEHMANN, J. R.; FORZZA, R. C.; SALINO, A.; COSTA, D. P. D. A.; SOBRAL, M.. Plantas da Floresta Atlântica. Rio de Janeiro. Rio de Janeiro: Jardim Botânico do Rio de Janeiro, 2009.

TROWBRIDGE, C. C.; STANLEY, A.; KAYE, T. N.; DUNWIDDIE, P. W.; WILLIAMS, J. L.. Long-term effects of prairie restoration on plant community structure and native population dynamics. Restoration Ecology, n.25, v.4, p.559- 
568, 2017. DOI: http://doi.org/10.1111/rec.12468

TURNER, I. M.; WONG, Y. K.; CHEW, P. T.; IBRAHIM, A. B.. Tree species richness in primary and old secondary tropical forest in Singapore. Biodivers Conserv, n.6, p.537-543, 1997

VALENTIN-SILVA, A.; GODINHO, M. A. S.; CRUZ, K. C.; LELIS, S. M.; VIEIRA, M. F.. Three psychophilous Asteraceae species with distinct reproductive mechanisms in southeastern Brazil. New Zealand Journal of Botany, n.54, p.498-510, 2016. DOI: http://doi.org/10.1080/0028825X.2016.1236735

VASCONCELOS, M. F.. O que são campos rupestres e campos de altitude nos topos de montanha do Leste do Brasil?. Revista Brasil. Bot., v.34, n.2, p.241-246, 2011.

VASCONCELOS, V. V.. Campos de altitude, campos rupestres e aplicação da lei da mata atlântica: estudo prospectivo para o estado de Minas Gerais. Boletim Geográfico, Maringá, v.32, n.2, p.110-133, 2014.

VERÍSSIMO, P. N.; SAFFORD, H. D. F.; BEHLING, H.. Holocene vegetation and fire history of the Serra do Caparaó, SE Brazil. Holocene, n.22, p.1243-1250, 2012. DOI: http://doi.org/10.1177/0959683612437864

VIANA, J. L.; BARBOSA, M. R. D. V.. Estrutura e composição do estrato herbáceo em um remanescente de Floresta Semidecidual Submontana no Nordeste do Brasil. Sitientibus - Série Ciências Biológicas, n.13, p.1-13, 2014. DOI: http://doi.org/10.13102/scb216

VILLAGRA, B. L. P.; GOMES, E. P. C.; BURNHAM, R. J.; ROMANIUC NETO, S.. Diversity and abundance of climbers from the Atlantic Forest, southeastern Brazil. Biodiversity and Conservation, n.22, p.2505-2517, 2013. DOI: http://doi.org/10.1007/s10531-013-0533-1

WEN, L.; DONG, S. K.; LI, Y. Y.; SHERMAN, R.; SHI, J. J.; LIU, D. $\mathrm{M}$.; ZHU, L.. The effects of biotic and abiotic factors on the spatial heterogeneity of alpine grassland vegetation at a small scale on the qinghai-tibet plateau (QTP), China. Environmental Monitoring and Assessment, n.185, p.80518064, 2013. DOI: http://doi.org/10.1007/s10661-013-3154-y

YOON, J.; CAO, X.; ZHOU, Q.; MA L. Q.. Accumulation of Pb, $\mathrm{Cu}$, and $\mathrm{Zn}$ in native plants growing on a contaminated Florida site. Sci Total Environ, n.368, p.456-464, 2006.

A CBPC - Companhia Brasileira de Produção Científica (CNPJ: 11.221.422/0001-03) detém os direitos materiais desta publicação. Os direitos referem-se à publicação do trabalho em qualquer parte do mundo, incluindo os direitos às renovações, expansões e disseminações da contribuição, bem como outros direitos subsidiários. Todos os trabalhos publicados eletronicamente poderão posteriormente ser publicados em coletâneas impressas sob coordenação da Sustenere Publishing, da Companhia Brasileira de Produção Científica e seus parceiros autorizados. Os (as) autores (as) preservam os direitos autorais, mas não têm permissão para a publicação da contribuição em outro meio, impresso ou digital, em português ou em tradução. 